\title{
CONFORMAL TRANSFORMATIONS AND CLIFFORD ALGEBRAS
}

\author{
PERTTI LOUNESTO AND ESKO LATVAMAA
}

\begin{abstract}
A spinor representation for the conformal group of the real orthogonal space $R^{p, q}$ is given. First, the real orthogonal space $R^{p, q}$ is compactified by adjoining a (closed) isotropic cone at infinity. Then the nonlinear conformal transformations are linearized by regarding the conformal group as a factor group of a larger orthogonal group. Finally, the spin covering group of this larger orthogonal group is realized in the Clifford algebra $R_{1+p, q}$ containing the Clifford algebra $R_{p, q}$ on the orthogonal space $R^{p, q}$. Explicit formulas for orthogonal transformations, translations, dilatations and special conformal transformations are given in Clifford language.
\end{abstract}

Introduction. We consider the real orthogonal space $R^{p, q}$ with the quadratic form

$$
x_{1}^{2}+x_{2}^{2}+\cdots+x_{p}^{2}-x_{p+1}^{2}-\cdots-x_{p+q}^{2},
$$

and construct a Clifford algebraic realization for the conformal group of $R^{p, q}$.

The following notations and facts are needed; for further details on Clifford algebras one may consult Chevalley [2], Atiyah-Bott-Shapiro [1] or Karoubi [4]. The universal Clifford algebra on $R^{p, q}$ is denoted by $R_{p, q}$ and the subspace of $k$-vectors by $R_{p, q}^{k}$.

By universality, the map $x \rightarrow-x$ in $X=R_{p, q}^{1}$ is uniquely extended to an automorphism $s \rightarrow \bar{s}$ and an antiautomorphism $s \rightarrow s^{-}$of $R_{p, q}$. Define the groups

$$
\begin{aligned}
\operatorname{Pin}(p, q) & =\left\{s \in R_{p, q} \mid s^{-} s= \pm 1, \text { and for all } x \in X \text { also } s x \bar{s}^{-1} \in X\right\}, \\
\operatorname{Spin}(p, q) & =R_{p, q}^{(0)} \cap \operatorname{Pin}(p, q),
\end{aligned}
$$

where $R_{p, q}^{(0)}$ is the even subalgebra of $R_{p, q}$. Then there are the exact sequences

$$
\begin{aligned}
& 1 \rightarrow Z_{2} \rightarrow \operatorname{Pin}(p, q) \stackrel{\rho}{\rightarrow} O(p, q) \rightarrow 1, \\
& 1 \rightarrow Z_{2} \rightarrow \operatorname{Spin}(p, q) \rightarrow S O(p, q) \rightarrow 1,
\end{aligned}
$$

the map $\rho$ being defined by $\rho(s) x=s x \bar{s}^{-1}$.

For the conformal group of $R^{p, q}$ we need a larger algebra: the Clifford algebra $R_{1+p, q}$. Consider $X=R_{p, q}^{1}$ as a subspace of $R_{1+p, q}$ formed by deleting from the canonical basis $e_{0}, e_{1}, e_{2}, \ldots, e_{p+q}$ of $R_{1+p, q}^{1}$ a vector $e_{0}, e_{0}^{2}=1$. The involution $s \rightarrow e_{0}^{-1} \bar{s} e_{0}$ of $R_{1+p, q}$ induces an interesting $Z_{2}$-grading of $R_{1+p, q}$ : it fixes the subalgebra $R_{p, q}$. Provide the subspace $W=R_{1+p, q}^{0}+R_{1+p, q}^{1}$ with the quadratic form $w \rightarrow w^{-} w$ making $W$ isomorphic to the orthogonal space $R^{1+q, 1+p}$. Every element of the special orthogonal group $S O_{c}(p, q)$ of $W$ can be represented in the

Received by the editors November 10, 1978.

AMS (MOS) subject classifications (1970). Primary 15A66, 53A50, 81A78; Secondary 53A30. 
form $w \rightarrow s w \bar{s}^{-1}$ where $s$ belongs to the group

$$
\operatorname{Spin}_{c}(p, q)=\left\{s \in R_{1+p, q} \mid s^{-} s= \pm 1 \text {, and for all } w \in W \text { also } s w \bar{s}^{-1} \in W\right\} \text {. }
$$

Since the map $w \rightarrow-\bar{w}$ in $W$ is a hyperplane reflection, we have a representation for the orthogonal group $O_{c}(p, q)$ of $W$ and also for its two-fold covering group $\operatorname{Pin}_{c}(p, q)$. It will be seen that $\operatorname{Pin}_{c}(p, q)$ is a four-fold (or double two-fold) covering group of the conformal group of $R^{p, q}$.

1. Conformal transformations. The real orthogonal space $R^{p, q}$ can be compactified by adjoining a (closed) isotropic cone at infinity. This compactification, called the Möbius space, is homeomorphic to $\left(S^{p} \times S^{q}\right) / Z_{2}$ and also to the real projective quadric $\mathscr{G}_{1}\left(R^{p+1, q+1}\right)$ (see Porteous [7] and for a detailed description of the case $p=1$, Segal [8]). The Möbius group acting on the Möbius space consists of sphere preserving transformations; it is generated by sphere inversions (which may look like hyperplane reflections in the underlying orthogonal space). The sphere preserving maps are also angle preserving and, excluding the low dimensional exceptions, they are the only angle preserving maps, see e.g. Haantjes [3].

In the orthogonal space $X$ the notions $x^{2}=\langle x, x\rangle$ and $x^{-1}=x\left(1 / x^{2}\right)$ if $x^{2} \neq 0$ are not symbolic abbreviations but well defined in the Clifford algebra. In the Möbius space $\check{X}$ which contains $X$ the inversion $x \rightarrow x^{-1}$ of the unit sphere interchanges the isotropic cone at the origin and the isotropic cone at infinity.

The conformal group is a two-component group or a four-component group according as $p q$ is even or odd. Every element in the identity component of the conformal group is a composition of one rotation, one translation, one dilatation and one special conformal transformation; so the maps

$$
\begin{aligned}
& x \rightarrow t x, \quad t \in S O_{+}(p, q), \\
& x \rightarrow x+y, \quad y \in X, \\
& x \rightarrow x \lambda, \quad \lambda \in R_{+}, \\
& x \rightarrow\left(x^{-1}+a\right)^{-1}, \quad a \in X,
\end{aligned}
$$

generate the identity component of the conformal group. The special conformal transformation $x \rightarrow\left(x^{-1}+a\right)^{-1}$ can be written in the form

$$
x \rightarrow\left(x+a x^{2}\right) \lambda(x), \quad \sigma(x)=1+2\langle a, x\rangle+a^{2} x^{2},
$$

where the scalar $\lambda(x)=(\sigma(x))^{-1}$ is the local magnification factor. This special conformal transformation is singular when $\sigma(x)=0$, i.e. in the cone $\left(x+a^{-1}\right)^{2}=$ 0 if $a^{2} \neq 0$, and in the hyperplane $\langle a, x\rangle=-\frac{1}{2}$ if $a^{2}=0$.

2. Conformal Lie algebra. The differential operators corresponding to rotations, translations, dilatations and special conformal transformations satisfy the commutation relations

$$
\begin{aligned}
{\left[M_{i j}, M_{k l}\right] } & =g_{i l} M_{j k}-g_{i k} M_{j l}+g_{j k} M_{i l}-g_{j l} M_{i k}, \\
{\left[P_{i}, M_{j k}\right] } & =g_{i j} P_{k}-g_{i k} P_{j}, \quad\left[D, P_{i}\right]=-P_{i}, \\
{\left[K_{i}, M_{j k}\right] } & =g_{i j} K_{k}-g_{i k} K_{j}, \quad\left[D, K_{i}\right]=K_{i}, \\
{\left[K_{i}, P_{j}\right] } & =2\left(g_{i j} D+M_{i j}\right) .
\end{aligned}
$$


It is well known that the rotation generators $M_{i j}=x_{i} \partial / \partial x^{j}-x_{j} \partial / \partial x^{i}$ can be realized as bivectors $\frac{1}{2} e_{i} e_{j}=\frac{1}{2} e_{i j}$. In order to obtain Clifford algebraic realizations for $P_{i}, D$ and $K_{i}$ we need a larger algebra: the Clifford algebra $R_{1+p, q}$.

Form the vector space $V=\frac{1}{2}\left(1+e_{0}\right) R_{p, q}^{0}+R_{p, q}$. Its bivector space $\frac{1}{2}\left(1+e_{0}\right) R_{p, q}^{1}$ $+R_{p, q}^{2}$ is isomorphic to the Lie algebra of the affine orthogonal group. The generators of rotations and translations can be realized as follows:

$$
M_{i j} \leftrightarrow \frac{1}{2} e_{i j}, \quad P_{i} \leftrightarrow-\frac{1}{2}\left(1+e_{0}\right) e_{i} .
$$

Similarly, the orthogonal space $W=R_{1+p, q}^{0}+R_{1+p, q}^{1}$ has the bivector space $R_{1+p, q}^{1}$ $+R_{1+p, q}^{2}$. As a Lie algebra, this bivector space is isomorphic to the Lie algebra of the conformal group. We find that the commutation relations (*) are satisfied by the elements given in Table 1 .

$$
\begin{array}{cl}
M_{i j} & \frac{1}{2} e_{i j} \\
P_{i} & -\frac{1}{2}\left(1+e_{0}\right) e_{i} \\
D & -\frac{1}{2} e_{0} \\
K_{i} & -\frac{1}{2}\left(1-e_{0}\right) e_{i}
\end{array}
$$

TABLE 1. Generators of conformal Lie algebra

Remark. The relativistic covariance of the Dirac field $\psi(x)$ is demonstrated by considering the translation expansion $\exp \left(y^{i} P_{i}\right) \psi(x)=\psi(x+y)$ together with the Lorentz covariance. Given an antisymmetric transformation $h$ in the Minkowski space, there is a Lorentz transformation $t=\exp (h)$. Furthermore, there is a unique bivector $b$ such that $h x=b x-x b$, namely $b=\frac{1}{2} b^{i j} e_{i j}$ where $b^{i j}=\frac{1}{2} h^{i j}$. By exponentiation $s=\exp (b)$ we then reach $t=\rho(s)$, i.e. $t x=s x s^{-1}$. The Lorentz covariance of the Dirac field $\psi(x)$ reads as follows:

$$
\exp \left(\frac{1}{2} b^{j i} e_{i j}\right) \exp \left(\frac{1}{2} h^{j i} M_{i j}\right) \psi(x)=s^{-1} \psi(t x)
$$

Here the angular momentum operator $M_{i j}$ and the spin operator $\frac{1}{2} e_{i j}$ act in opposite directions $-a$ consequence of the fact that only the total angular momentum is a constant of motion.

3. Covering group of conformal group. We shall construct a set $T$ and a map $w$ : $X \rightarrow T$ so that conformal transformations $f$ of $X$ can be represented as maps $w(x) \rightarrow w(f(x)) \sigma(x)=s w(x) \bar{s}^{-1}$ possibly followed by a map $w \rightarrow-\bar{w}$. Here the scalar $\lambda(x)=(\sigma(x))^{-1}$ is the local magnification factor.

Consider first translations $x \rightarrow x+y, y \in X$. Then we have

$$
s=\exp \left(\frac{1}{2}\left(1+e_{0}\right) y\right)=1+\frac{1}{2}\left(1+e_{0}\right) y \text {. }
$$

As a first trial we compute the action of $s$ on $V=\frac{1}{2}\left(1+e_{0}\right) R_{p, q}^{0}+R_{p, q}^{1}$. For $x \in X$ we set $v(x)=\frac{1}{2}\left(1+e_{0}\right) x^{2}+x \in V$. Then

$$
\begin{aligned}
s v(x) \bar{s}^{-1} & =\left(1+\frac{1}{2}\left(1+e_{0}\right) y\right)\left(\frac{1}{2}\left(1+e_{0}\right) x^{2}+x\right)\left(1+\frac{1}{2}\left(1-e_{0}\right) y\right) \\
& =\frac{1}{2}\left(1+e_{0}\right)\left(x^{2}+2\langle x, y\rangle\right)+x \neq v(x+y) .
\end{aligned}
$$


Hence this construction is not successful. Since

$$
\operatorname{ses}^{-1}=\frac{1}{2}\left(1+e_{0}\right) y^{2}+y+\frac{1}{2}\left(1-e_{0}\right) \text { for } e=\frac{1}{2}\left(1-e_{0}\right)
$$

we define

$$
\begin{aligned}
T & =\frac{1}{2}\left(1+e_{0}\right) R_{p, q}^{0}+R_{p, q}^{1}+\frac{1}{2}\left(1-e_{0}\right), \\
w(x) & =\frac{1}{2}\left(1+e_{0}\right) x^{2}+x+\frac{1}{2}\left(1-e_{0}\right)
\end{aligned}
$$

where $w(x) \in T$. So in $T$ we have the formula for translations $s w(x) \bar{s}^{-1}=$ $w(x+y)$.

Obviously $T$ is not a vector subspace of $W=R_{1+p, q}^{0}+R_{1+p, q}^{1}$ but $T$ is an affine subspace. An element $w=w(x)$ in $W$ is isotropic for all $x \in X$, i.e. $w^{-} w=0$. Since the Möbius space $\check{X}$ is homeomorphic to $g_{1}\left(R^{p+1, q+1}\right)$, we can identify $\check{X}$ with the set of the equivalence classes $\{w \sigma \mid \sigma \in \hat{R}\}, w \in W, w \neq 0, w^{-} w=0$, where $\hat{R}$ consists of the nonzero real numbers, and let the conformal group $G(p, q)$ of $X$ act on this set.

THEOREM 1. Every conformal transformation $f$ of $X$ can be written in the form

$$
w(x) \rightarrow w(f(x)) \sigma(x)=\left\{\begin{array}{l}
s w(x) \bar{s}^{-1}, \\
-\bar{s} \bar{w}(x) s^{-1}
\end{array}\right.
$$

where $s \in \operatorname{Spin}_{c}(p, q)$.

Proof. We list explicit formulas for a sufficient set of generating conformal transformations.

Rotations. $x \rightarrow t x, t=\exp (h), s=\exp \left(-\frac{1}{2} b^{j i} e_{i j}\right)$.

$$
s w(x) \bar{s}^{-1}=\frac{1}{2}\left(1+e_{0}\right) x^{2}+s x s^{-1}+\frac{1}{2}\left(1-e_{0}\right)=w(t x) .
$$

Translations. $x \rightarrow x+y, s=\exp \left(\frac{1}{2}\left(1+e_{0}\right) y\right)=1+\frac{1}{2}\left(1+e_{0}\right) y$.

$s w(x) \bar{s}^{-1}=\frac{1}{2}\left(1+e_{0}\right)\left(x^{2}+2\langle x, y\rangle+y^{2}\right)+x+y+\frac{1}{2}\left(1-e_{0}\right)=w(x+y)$.

Dilatations. $x \rightarrow x \lambda, \lambda=\exp (\eta), s=\exp \left(\frac{1}{2} e_{0} \eta\right)$.

$$
s w(x) \bar{s}^{-1}=\frac{1}{2}\left(1+e_{0}\right) x^{2} \lambda+x+\frac{1}{2}\left(1-e_{0}\right) \lambda^{-1}=w(x \lambda) \sigma, \quad \sigma=\lambda^{-1} .
$$

Special conformal transformations. $x \rightarrow\left(x^{-1}+a\right)^{-1}=\left(x+a x^{2}\right) \lambda(x)$, $\sigma(x)=1+2\langle a, x\rangle+a^{2} x^{2}, s=\exp \left(\frac{1}{2}\left(1-e_{0}\right) a\right)=1+\frac{1}{2}\left(1-e_{0}\right) a$.

$$
\begin{aligned}
s w(x) \bar{s}^{-1} & =\frac{1}{2}\left(1+e_{0}\right) x^{2}+x+a x^{2}+\frac{1}{2}\left(1-e_{0}\right) \sigma(x) \\
& =w\left(\left(x+a x^{2}\right) \lambda(x)\right) \sigma(x), \text { when } \sigma(x) \neq 0 .
\end{aligned}
$$

Inversion. $x \rightarrow-x^{-1}\left(x^{2} \neq 0\right)$.

$$
\begin{aligned}
-\bar{w}(x) & =-\frac{1}{2}\left(1-e_{0}\right) x^{2}+x-\frac{1}{2}\left(1+e_{0}\right) \\
& =w\left(-x^{-1}\right) \sigma(x)=w(x \lambda(x)) \sigma(x), \quad \sigma(x)=-x^{2} .
\end{aligned}
$$

Orthogonal transformation $t \in O(p, q) \backslash S O(p, q)$.

$$
\begin{aligned}
s w(x) \bar{s}^{-1} & =-\frac{1}{2}\left(1-e_{0}\right) x^{2}+t x-\frac{1}{2}\left(1+e_{0}\right) \\
& =w(t x \lambda(x)) \sigma(x), \quad \sigma(x)=-x^{2} .
\end{aligned}
$$


We have the commutative diagram

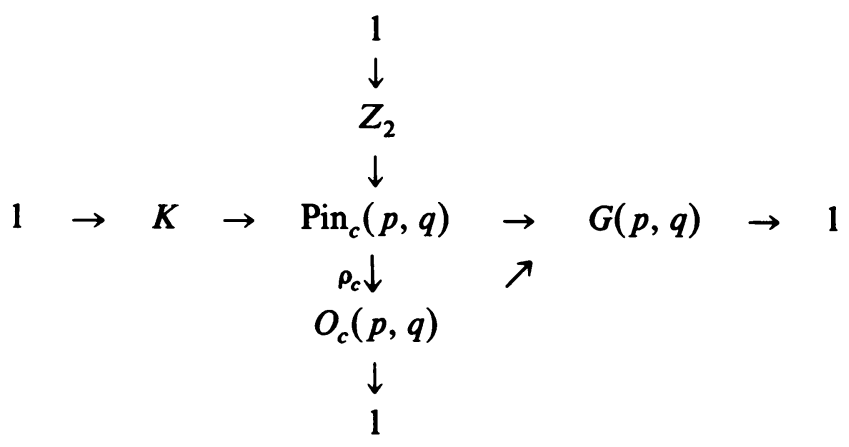

of exact sequences. In the covering group $\operatorname{Pin}_{c}(p, q)$ of the conformal group $G(p, q)$ the preimage of the identity is $K=\left\{1,-1, e_{J},-e_{J}\right\}$ where $e_{J}=$ $e_{0} e_{1} e_{2} \cdots e_{p+q}$

It is amusing to note that $w(x)$ factorizes as $w(x)=(1+x) e(1+x)$ where $e=\frac{1}{2}\left(1-e_{0}\right)$ is an idempotent.

The map $w(x) \rightarrow s w(x) \bar{s}^{-1}$ has the following geometric interpretation. First, the image set of the map $x \rightarrow w(x)$ is the intersection of the hyperplane $T$ and the isotropic cone in $W$. In rotations and translations this set is mapped onto itself. In a dilatation $x \rightarrow x \lambda$ we have $w(x) \rightarrow w(x \lambda) \sigma$ and $T$ is moved to $T \sigma$. In a special conformal transformation $x \rightarrow\left(x^{-1}+a\right)^{-1}$ the hyperplane $T$ is tilted so that it becomes perpendicular to the vector $w\left(a^{-1}\right)$ while the point $w(0)$ is kept fixed.

It is interesting to have a general formula for the reciprocal $\sigma(x)$ of the local magnification factor $\lambda(x)$. Denote $u^{+}=(\bar{u})^{-}$and $e=\frac{1}{2}\left(1-e_{0}\right)$.

TheOREM 2. (i) If $w(f(x)) \sigma(x)=s w(x) \bar{s}^{-1}$ define $v=e s(1+x) e$ and $u=v+\bar{v}$. Then $\sigma(x)= \pm u^{+} u$ according as $s^{-} s= \pm 1$.

(ii) If $w(f(x)) \sigma(x)=-\bar{s} \bar{w}(x) s^{-1}$ define $v=\bar{e} s(1+x) e$ and $u=v-\bar{v}$. Then $\sigma(x)= \pm u^{+} u$ according as $s^{-} s=\mp 1$.

2.

Proof. Direct calculations, recalling that $w(x)=(1+x) e(1+x)$, lead to Table

$$
\begin{array}{llll} 
& \multicolumn{1}{c}{f(x)} & \multicolumn{1}{c}{u(x)} & \multicolumn{1}{c}{\sigma(x)} \\
\text { (i) } & s x s^{-1} & s & 1 \\
& x+y & 1 & 1 \\
& x \exp (\eta) & \exp (-1 / 2 \eta) & \exp (-\eta) \\
& \left(x^{-1}+a\right)^{-1} & 1+a x & 1+2\langle a, x\rangle+a^{2} x^{2} \\
\text { (ii) } & -\left(s x s^{-1}\right)^{-1} & s x & -x^{2} \\
& -(x+y)^{-1} & x+y & -(x+y)^{2} \\
& -x^{-1} \exp (-\eta) & x \exp (1 / 2 \eta) & -x^{2} \exp (\eta) \\
& -\left(x^{-1}+a\right) & x & -x^{2}
\end{array}
$$

TABLE 2. Local magnification factor 
The significance of Theorem 2 is that the elements $u=u(x)$, in a sense square roots of $\sigma(x)$, can be used to study the transformation properties of conformally covariant spinor fields. Under the conformal transformations $x \rightarrow f(x)$ of the Minkowski space the Dirac spinor field $\psi(x)$ of conformal degree $3 / 2$ is transformed into $u^{-1} \psi(f(x)) \lambda(x)$ or $-\bar{u}^{-1} \bar{\psi}(f(x)) \lambda(x)$. These notions generalize some of the results in two papers of Kastrup [5], [6].

\section{REFERENCES}

1. M. F. Atiyah, R. Bott and A. Shapiro, Clifford modules, Topology 3 (1964), 3-38.

2. C. Chevalley, The algebraic theory of spinors, Columbia Univ. Press, New York, 1954.

3. J. Haantjes, Conformal representations of an $n$-dimensional euclidean space with a non-definite fundamental form on itself, Nederl. Akad. Wetensch. Proc. 40 (1937), 700-705.

4. M. Karoubi, K-theory, Springer, Berlin-Heidelberg-New York, 1978.

5. H. A. Kastrup, Zur physikalischen Deutung und darstellungstheoretischen Analyse der konformen Transformationen von Raum und Zeit, Ann. Physik (7) 9 (1962), 388-428.

6. , Some experimental consequences of conformal invariance at extremely high energies, Phys. Letters 3 (1962), 78-80.

7. I. Porteous, Topological geometry, Van Nostrand-Reinhold, London, 1969.

8. I. E. Segal, Mathematical cosmology and extragalactic astronomy, Academic Press, New York-San Francisco-London, 1976.

Institute of Mathematics, Helsinki University of Technology, SF-02150 Otaniemi, Finland

Department of Mathematics, University of JoensuU, 80120 JoensuU 12, FinLand 